(1) BIOPSYCHOSOCIAL
MEDICINE

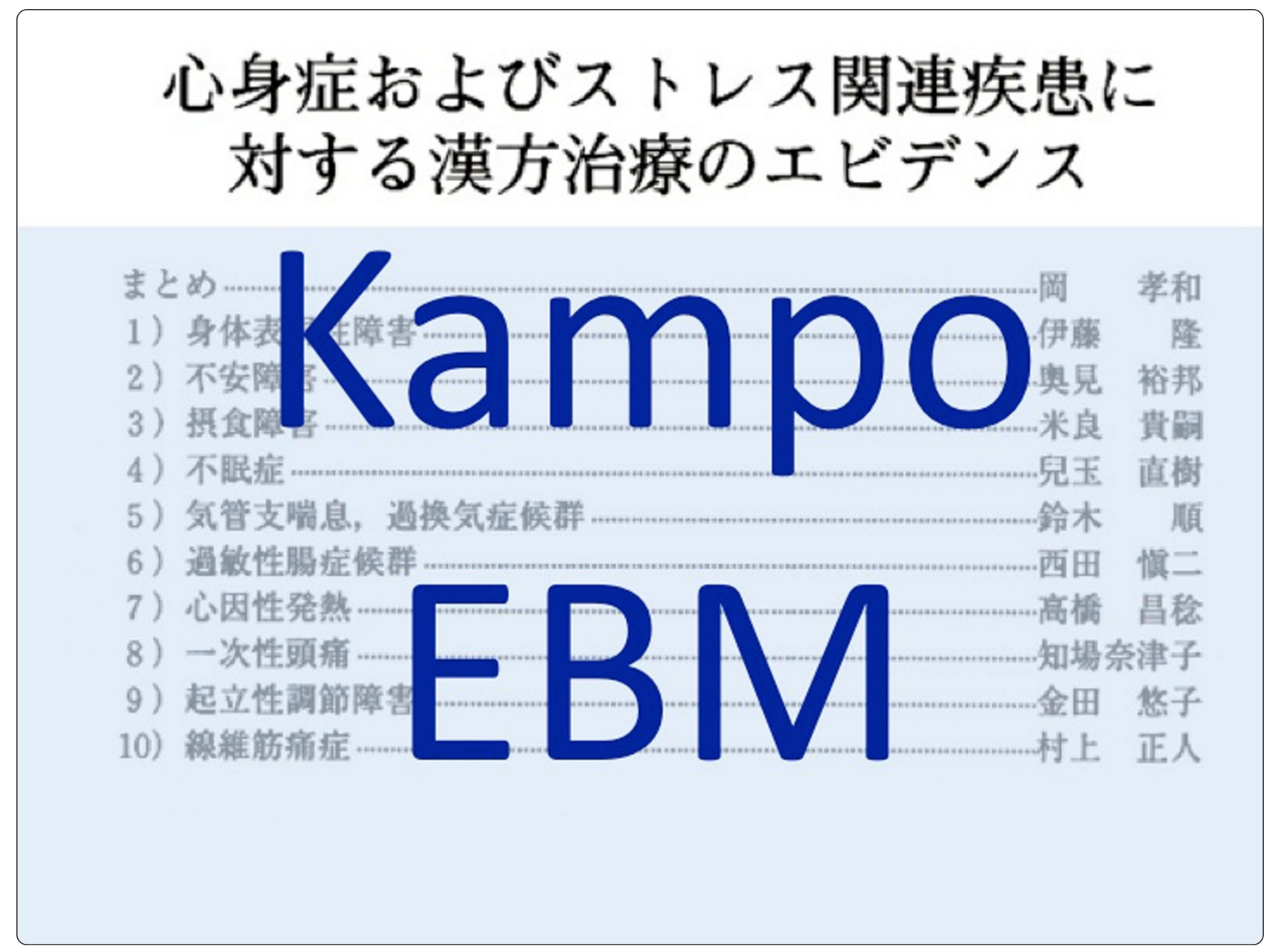

\title{
Effects of Kampo on functional gastrointestinal disorders
}

Oka et al.

C Biomed Central 


\title{
Effects of Kampo on functional gastrointestinal disorders
}

\author{
Takakazu Oka ${ }^{1 *}$, Hirokuni Okumi ${ }^{2}$, Shinji Nishida ${ }^{3}$,Takashi Ito ${ }^{4}$, Shinichi Morikiyo ${ }^{5}$, Yoko Kimura ${ }^{6}$, \\ Masato Murakami ${ }^{7}$ and JOPM-EBM Working Team
}

\begin{abstract}
This article reviews the effectiveness of Kampo (traditional Japanese herbal medicine) in the treatment of functional gastrointestinal disorders, especially functional dyspepsia (FD) and irritable bowel syndrome (IBS). The results of four randomized, controlled trials (RCTs) suggested the usefulness of rikkunshito in relieving the subjective symptoms of patients with FD. Rikkunshito significantly improved not only gastric symptoms, such as epigastiric discomfort, but also extra-gastric symptoms, such as general fatigue, when compared with control drugs. The therapeutic effects of rikkunshito were more evident when it was prescribed to patients with "kyosho", i.e., low energy. Two RCTs suggested the efficacy of keishikashakuyakuto for IBS.

Basic research studies have demonstrated that these Kampo medicines have multiple sites of action to improve subjective symptoms. For example, rikkunshito improves gastric motility dysfunction, including impaired adaptive relaxation and delayed gastric emptying, gastric hypersensitivity, and anorexia via facilitation of ghrelin secretion. It also exhibits anti-stress effects, i.e., it attenuates stress-induced exacerbation of gastric sensation and anorexia, as well as the hypothalamic-pituitary-adrenocortical axis and sympathetic activation. Keishikashakuyakuto exhibited not only an antispasmodic effect on intestinal smooth muscle, but also antidepressant-like effects. Case series suggest that other Kampo prescriptions are also effective for FD and IBS. However, further studies are necessary to evaluate their efficacy.
\end{abstract}

Keywords: Kampo, Rikkunshito, Keishikashakuyakuto, Functional dyspepsia, Irritable bowel syndrome

Kampo refers to Japanese traditional medicine, including herbal medicine. In Japan, herbal medicine is very popular, and it is covered by national health insurance. Psychosomatic medicine specialists often prescribe Kampo medicines to treat functional somatic diseases, such as functional gastrointestinal disorders (FGIDs). Patients with FGIDs complain of symptoms originating from the gastrointestinal tract without evident organic diseases. These symptoms are bothersome for patients and difficult to treat, thus decreasing patient quality of life. The pathophysiology of FGIDs is multifactorial. Both physiological factors, including abnormal motility, visceral

\footnotetext{
*Correspondence: oka-t@cephal.med.kyushu-u.ac.jp

'Department of Psychosomatic Medicine, Graduate School of Medical

Sciences, Kyushu University, Fukuoka 812-8582, Japan

Full list of author information is available at the end of the article
}

hypersensitivity, inflammation, and altered bacterial flora, as well as psychosocial factors, including psychological stress, psychological state, coping style, and social support affect brain-gut interactions and clinical outcomes [1,2].

Currently, many Kampo formulas are prescribed to treat FGIDs. However, the evidence of their efficacy is limited. Furthermore, as many earlier studies conducted in the 1980's and 90's were published in Japanese, those pioneering works have not been introduced into the English literature or indexed in PubMed.

Therefore, the aims of this paper are (1) to review the clinical trials that investigated the effect of Kampo on FGIDs, including studies published in Japanese, and (2) to provide an overview of the basic mechanisms as to how Kampo improves the subjective symptoms of patients with FGIDs, focusing on functional dyspepsia (FD) and irritable bowel syndrome (IBS).

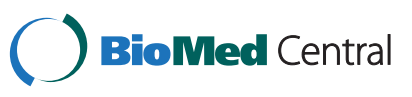




\section{Methods}

We performed literature searches on PubMed and Ichushi Web (papers written in Japanese) on June 30, 2013 using the keywords Kampo (or each prescription name) and functional dyspepsia or irritable bowel syndrome. We included reports regarding Kampo extract products that comply with the formulation standards established in 1986 and excluded those regarding drug solutions of crude drug pieces for decoction, powdered crude drugs, and over the counter (OTC) products. We abstracted clinical studies conducted as randomized, controlled trials (RCTs) and case series that involved at least 10 cases. We present the results descriptively, because a meta-analysis was not possible due to the limited number of RCTs. We also introduce basic research findings to explain the possible mechanisms of action of each Kampo medicine.

\section{Results}

\section{Functional dyspepsia}

To date, four RCTs, including one double blind (DB)RCT and 13 case series have been published. All studies were conducted in Japan. Most of them, especially all of the RCTs, studied the effectiveness of rikkunshito on dyspeptic symptoms. In these studies, the diagnosis of FD was based on either the American Gastroenterology Association working party criteria, in which the condition is named non-ulcer dyspepsia [3], or the Rome III diagnostic criteria, in which the condition is named FD [4]. In this analysis, we included both.

\section{Efficacy of rikkunshito}

According to the traditional medicine point of view, rikkunshito is a Kampo medicine for treating upper gastrointestinal symptoms, such as nausea, indigestion, and anorexia in patients with "kyo-sho", i.e., subjects who have weak constitutions and low vital energy. Rikkunshito extract granules for ethical use (Tsumura and Co., Product number TJ-43) (7.5 g) contain $4.0 \mathrm{~g}$ of dried extract obtained from mixed raw herbs in the following ratio: Atractylodes Lancea Rhizome, 4.0 g; Ginseng, 4.0 g; Pinellia Tuber, 4.0 g; Poria Sclerotium, 4.0 g; Jujube, 2.0 g; Citrus Unshiu Peel, 2.0 g; Glycyrrhiza, 1.0 g; and Ginger, $0.5 \mathrm{~g}$.

Miyoshi and colleagues conducted the first multi-center RCT to compare the therapeutic effects of rikkunshito and cisapride, a prokinetic drug, on the digestive symptoms of patients with non-ulcer dyspepsia. Subjects were randomized to receive either oral treatment with $2.5 \mathrm{~g}$ rikkunshito three times daily (rikkunshito group, 111 subjects) or cisapride $(2.5 \mathrm{mg}$ ) three times daily (cisapride group, 104 subjects) for four weeks. Significantly greater improvement of upper gastrointestinal symptoms, especially epigastralgia and abdominal discomfort, was found for rikkunshito than for cisapride [5]. Rikkunshito also resulted in greater, but non-significant, improvement of extra-gastric symptoms, such as coldness in the lower extremities, dizziness, and appetite loss when compared with cisapride. The global improvement rate of the rikkunshito group was higher than that of the cisapride group when this Kampo medicine was prescribed to subjects whose constitutions were weak or who felt moderate general fatigue, i.e., subjects with "kyo-sho".

Harasawa and coworkers conducted a multi-center DB$\mathrm{RCT}$ to assess the effect of rikkunshito on dismotility-like dyspepsia [6], one subtype of FD defined by the Rome II diagnostic criteria. Subjects were randomized to receive either oral treatment with $2.5 \mathrm{~g}$ rikkunshito three times daily (rikkunshito group, 133 subjects) or 2.5 -g granules containing a low dose $(2.5 \%)$ of rikkunshito three times daily as a control (control group, 117 subjects) for two weeks. Rikkunshito significantly improved the dysmotilitylike dyspepsia generalized improvement rate (DDGIR) and subjective symptoms, including epigastric discomfort, anorexia and, interestingly, general fatigue [7] when compared with the control group. In the rikkunshito group, the DDGIR improved more in patients whose vigor or physical strength was low when compared with those whose energy or strength was normal. The incidence of adverse events was not different between the two groups, i.e., adverse events were reported by seven subjects in the rikkunshito group and seven subjects in the control group. The most frequently observed adverse symptom was diarrhea (3 subjects in each group). The results of these two RCTs suggested the effectiveness of rikkunshito for FD. Furthermore, these studies also suggested that rikkunshito is more effective when it is prescribed to subjects with "kyo-sho", based on the traditional medicine diagnosis in patients with FD. These findings were published in Japanese.

Tatsuta and colleagues investigated the effects of rikkunshito on gastric emptying as well as the gastrointestinal symptoms of 42 dyspeptic patients in a RCT and first reported their findings in English [8]. In this study, by random allocation, 22 patients received oral treatment with $2.5 \mathrm{~g}$ rikkunshito three times daily, and $20 \mathrm{pa}-$ tients received combizym as a placebo. Gastric emptying was measured by the acetaminophen absorption method. After seven days of treatment, gastric emptying was significantly accelerated, and gastrointestinal symptoms, such as epigastric fullness, heartburn, belching, and nausea, were significantly improved in patients treated with rikkunshito. In contrast, placebo treatment produced no significant effects on gastric emptying and subjective symptoms.

Arai and colleagues compared the effects of four weeks of treatment with rikkunshito or domperidone, a prokinetic drug, on the gastrointestinal symptoms of 27 patients with FD in a RCT and found that dyspeptic symptoms improved significantly in both groups [9]. 
The results of these four RCTs indicate that rikkunshito is a useful drug for treating FD. Two RCTs suggested that the therapeutic effect of rikkunshito on FD was stronger when it is prescribed to subjects with "kyo-sho", based on the traditional medicine diagnosis. There were 13 case series. Most of them reported the effectiveness of rikkunshito. Other studies suggested the effectiveness of hangekobokuto $[10,11]$ and hangeshashinto. Notably, two studies suggested that hangeshashinto is effective for dyspeptic patients for whom in rikkunshito was not effective $[12,13]$.

\section{Therapeutic mechanisms of rikkunshito}

The subjective symptoms of FD are attributed to disturbed gastric motility and gastric hypersensitivity [4,14]. Psychological stress affects gastric motility [15] and gastric sensitivity $[16,17]$ and causes or exacerbates FD symptoms [18]. Some patients with FD suffer from co-morbid psychiatric disorders, such as depressive and anxiety disorders [18-20]. Any or all of these components may contribute to the development of subjective symptoms in patients with FD $[20,21]$. Human and animal studies have shown that rikkunshito has effects on each of these factors.

\section{Gastric motility dysfunction}

In patients with FD, gastric motility functions, such as gastric adaptive relaxation (or gastric accommodation) and gastric emptying, are impaired.

\section{Gastric emptying}

Harasawa and coworkers have demonstrated that a twoweek administration of rikkunshito accelerated gastric emptying, as assessed by the acetaminophen absorption method, and improved upper gastrointestinal symptoms in patients with FD whose gastric emptying was delayed [22]. Tatsuta and coworkers also reported that rikkunshito improved gastric emptying in patients with FD [8]. Animal studies have suggested that rikkunshito accelerates gastric emptying $[8,22]$ via nitric oxide-mediated mechanisms [23] and antagonism of 5-HT3 receptors [24].

\section{Adaptive relaxation}

Two weeks of rikkunshito administration significantly expanded the proximal stomach after incremental ingestion of a liquid meal up to $400 \mathrm{~mL}$ in patients with FD, suggesting that rikkunshito promotes gastric adaptive relaxation [25], a finding that had been observed in animal models [26,27].

\section{Other motility functions}

Rikkunshito was also reported to coordinate gastric myoelectric activity in patients with dyspeptic symptoms after gastrointestinal surgery [28] and to stimulate gastrointestinal motility in dogs $[29,30]$.

\section{Gastric hypersensitivity}

The effect of rikkunshito on gastric hypersensitivity has not yet been investigated in patients with FD. However, in healthy subjects, rikkunshito was reported to reduce epigastric fullness following gastric distension via a gastric barostat. Rikkunshito also attenuated psychological stress-induced reduction of gastric volume at the sensory threshold and stress-induced increased anxiety at the discomfort threshold [31], suggesting that rikkunshito improves gastric symptoms under distension, possibly by affecting gastric sensorimotor function.

\section{Ghrelin secretion}

Ghrelin is a 28-amino acid orexigenic peptide found in the stomach [32]. Takeda and colleagues demonstrated that rikkunshito suppresses cisplatin-induced decreases in plasma levels of acylated ghrelin, an active form of ghrelin, and that it increases food intake via serotonin (5-HT) $2 \mathrm{~B} / 2 \mathrm{C}$ receptor antagonism in rats [33]. After that report, research intensified to determine whether rikkunshito increases ghrelin secretion in human subjects, leading to improvement of appetite and dyspeptic symptoms (for review, see [34]). Oral administration of rikkunshito for two or four weeks has been demonstrated to increase plasma levels of acylated ghrelin in healthy subjects [35], patients with gastric cancer [36], and patients with FD [9]. Arai and coworkers compared the effects of rikkunshito with the effect of domperidone on plasma ghrelin levels and gastrointestinal symptoms in patients with FD. Four weeks after treatment, the plasma level of acylated ghrelin in the rikkunshito group was significantly higher than that in the domperidone group, whereas the plasma level of desacylated ghrelin, an inactive form of ghrelin, was not different between the two groups [9]. Furthermore, in the rikkunshito group, the improvements in reflux and indigestion showed positive correlations with the increase in acylated ghrelin. Among the eight ingredients of rikkunshito, flavonoids of citrus unshiu peel, i.e., heptamethoxyflavone and hesperidin, and flavonoids of glycyrrhiza, i.e., isoliquiritigenin have $5-\mathrm{HT}_{2 \mathrm{~B}}$ antagonist activity and reverse the cisplatininduced suppression of plasma acylated ghrelin [33]; and atractylodin, a component of the Atractylodes Lancea Rhizome, potentiates ghrelin receptor signaling [37].

These findings suggest that rikkunshito improves the subjective symptoms of FD, at least in part, by facilitating the secretion of ghrelin.

\section{Psychological factors and stress}

We assessed the effect of 4 weeks of rikkunshito administration on depressive symptoms in patients with FD. Rikkunshito significantly decreased depression scores, as assessed by the Self-Rating Questionnaire for Depression [38]. As depressed patients frequently show high serum 
cortisol levels, we also investigated the effect of four weeks of rikkunshito administration on serum cortisol levels at 9 a.m. in patients with FD [39]. Seven of 23 patients showed levels higher than the normal range. Four weeks of rikkunshito administration significantly decreased their cortisol levels, whereas rikkunshito did not affect the cortisol levels of patients with levels in the normal range. An animal study has demonstrated that rikkunshito attenuates the activities of corticotropinreleasing hormone (CRH)-producing neurons and reduces anxiety-like behavior in tumor-bearing rats. As cancerinduced anorexia and cachexia are induced by $5-\mathrm{HT}_{2 \mathrm{c}}$ receptor-mediated interactions of 5-HT with $\mathrm{CRH}$ producing neurons and $\mathrm{CRH}$ activates the hypothalamicpituitary-adrenocortical (HPA) axis, decreases the plasma levels of acylated ghrelin, and induces anxiety-like behavior, the inhibitory effect of rikkunshito on $\mathrm{CRH}$-producing neurons may contribute to psychological effects and normalization of high cortisol levels [37].

Rikkunshito was also shown to attenuate stress-induced physiological responses. For example, rikkunshito attenuated acute stress-induced increases in plasma levels of adrenocorticotropic hormone, cortisol [40], and neuropeptide Y [41] evoked by repeated blood sampling in healthy subjects. As neuropeptide $\mathrm{Y}$ and noradrenaline are coreleased from the sympathetic nerve terminals by activation of the sympathetic nervous system and are released into plasma during intensive sympathetic activation, neuropeptide $\mathrm{Y}$ is considered a marker of stress-induced sympathetic activation.

In addition to its inhibitory effect on the stress-induced activation of the HPA axis and the sympathetic nervous system, rikkunshito improved the delayed gastrointestinal tract motility induced by stress mimicking a Japanese summer, i.e., high temperature and humidity stress in mice [42]. Rikkunshito also reversed the stress-induced reduction of intragastric volume stimulated by a barostat at the sensory threshold in healthy subjects [31]. Furthermore, rikkunshito reversed the novelty stress-induced reduction in food intake via ghrelin secretion in rats [43]. Based on these findings, "A Guideline for the Diagnosis and Treatment of Psychosomatic Diseases 2006" recommends prescription of rikkunshito for treating FD [44]. Furthermore, rikkunshito is widely prescribed for treating the upper gastrointestinal tract symptoms of patients with gastroesophageal reflux disease [45-47], gastric cancer [36], patients who underwent gastrointestinal surgery [28] or chemotherapy $[48,49]$, and patients who take selective serotonin reuptake inhibitors [50]. Rikkunshito is also thought to be a promising drug for ameliorating anorexiacachexia in patients with cancer [37].

\section{Irritable bowel syndrome}

To date, two RCTs, including one DB-RCT and 14 case series have been published. All studies were conducted in Japan and published in Japanese. Two RCTs studied the effectiveness of keishikashakuyakuto on IBS [51,52].

\section{Efficacy of keishikashakuyakuto}

Keishikashakuyakuto is a Kampo medicine that is prescribed to relieve abdominal pain. Keishikashakuyakuto extract granules for ethical use (Tsumura and Co., Product number TJ-60) (7.5 g) contain $3.75 \mathrm{~g}$ of dried extract obtained from mixed raw herbs in the following ratio: Peony root, 6.0 g; Cinnamon Bark, 4.0 g; Jujube Fruit, 4.0 g; Glycyrrhiza Root, 2.0 g; and Ginger Rhizome, $1.0 \mathrm{~g}$.

Mizuno and colleagues conducted the first RCT to compare the therapeutic effect of keishikashakuyakuto with mepenzolate bromide, an anti-cholinergic agent, on IBS. Subjects were randomized to receive either oral treatment with $2.5 \mathrm{~g}$ keishikashakuyakuto three times daily (keishikashakuyakuto group, 26 subjects) or mepenzolate bromide (15 mg) three times daily (mepenzolate group, 24 subjects) for eight weeks. At four weeks after the start of treatment, the keishikashakuyakuto group had significantly greater improvement than mepenzolate of IBS symptoms, including stool abnormality, abdominal pain, gas symptoms, and rumbling stomach. The final overall improvement rate was significantly higher in the keishikashakuyakuto group than in the mepenzolate group [51].

Sasaki and coworkers conducted a multi-center DBRCT to investigate the effect of keishikashakuyakuto. Subjects were randomized to receive either oral treatment with $2.0 \mathrm{~g}$ keishikashakuyakuto (EK-60, Kracie and Co.) three times daily (keishikashakuyakuto group, 124 subjects) or 2.0-g granules containing a low dose (5\%) of keishikashakuyakuto three times daily (low-dose group, 108 subjects) for eight weeks. The final overall improvement rate was not different between the two groups. However, the percentage of patients with improved abdominal pain was significantly higher in the keishikashakuyakuto group (58\% improved moderately or more) than in the low-dose group (37\%) in patients with the diarrhea-predominant type IBS. Such a difference was not found in patients with the constipation-predominant type or alternating type IBS. This study suggests that keishikashakuyakuto is effective for relieving abdominal pain in patients with the diarrhea-predominant type IBS [52].

Among 14 case studies of IBS, most studied the effectiveness of keishikashakuyakuto. Others investigated the effectiveness of other Kampo formulas and suggested their usefulness. Such prescriptions included saireito, keihito, heiisan, and daikenchuto. Oka and colleagues found that, in spite of favorable improvement of abdominal symptoms such as abdominal pain or diarrhea (percentage of moderate improvement or more two weeks after treatment, $>80 \%$ ), keishikashakuyakuto was less effective for 
extra-colonic symptoms, such as general fatigue or appetite loss (50-60\%). They demonstrated that Kampo therapy became more effective for relieving the extra-colonic symptoms of IBS patients who did not respond to keishikashakuyakuto when prescribed based on the traditional medicine diagnosis, considering the dimensions of "ki-ketsu-sui" or "coldness-heat" balances [53].

\section{Therapeutic mechanisms of keishikashakuyakuto}

Peony root and Glycyrrhiza root, both of which are the main crude ingredients of keishikashakuyakuto, suppress the neurogenic contractions of the ileum induced by electrical stimulation and ganglionic-stimulating agents, such as dimethylphenylpiperazinium and nicotine in guinea pigs via inhibition of acetylcholine (Ach) release from cholinergic nerves and inhibition of Ach action on ileum smooth muscle [54]. Their antispasmodic effect was also confirmed for the human colon [55]. In addition to its spasmolytic and smooth muscle-relaxing effects [56-58], peony root has anti-inflammatory and analgesic effects and inhibits gastric acid secretion and stressinduced ulceration [59]. Furthermore, peony root was demonstrated to have sedative [60] and antidepressantlike effects in mice [61-66]. The antidepressant-like effect of peony root could be, at least in part, mediated by inhibiting monoamine oxidase activity, hypothalamicpituitary-adrenal axis activation, oxidative stress, and upregulated brain-derived neurotrophic factor [62-66].

\section{Discussion}

We reviewed RCTs that evaluated the effects of Kampo medicines on FGIDs. Clinical trial results have suggested that several Kampo medicines are useful for relieving the subjective symptoms of patients with FGIDs, i.e., rikkunshito for FD and keishikashakuyakuto for IBS.

In addition to efficacy, these studies suggest several interesting characteristics of Kampo as pharmacotherapy. First, rikkunshito was effective not only for gastrointestinal symptoms but also for the extra-gastric symptoms of patients with FD, such as dizziness, coldness in the lower extremities, or general fatigue. As patients with
FGIDs are known to complain of many symptoms that are not of gastrointestinal origin $[67,68]$, this characteristic of Kampo medicine may be beneficial for improving the quality of life of these patients. Second, basic research has demonstrated that Kampo medicines have multiple sites of action to alleviate symptoms. As was described previously, Kampo medicines act on both the central nervous system (mind) and the gastrointestinal tract (body) to relieve symptoms. They also exhibit antistress effects. These multiple actions could be beneficial for patients with FGID and comorbid psychiatric disorders, patients whose physical symptoms are modulated by psychological factors such as anxiety or depression, or patients whose symptoms are exacerbated by psychological stress (Table 1). Third, Kampo medicines, at least rikkunshito and keishikshakuyakuto, become more effective if patients are diagnosed and treated based on the viewpoint of traditional medicine. This is reasonable because Kampo medicines were originally prescribed based on a traditional medicine diagnosis, e.g. "kyo-sho", not on the modern medicine diagnosis, e.g. FD. To accumulate evidence for the efficacy of Kampo medicine, it is necessary to conduct RCTs to evaluate its efficacy based on modern medicine diagnoses. In the future, however, clinical studies should be conducted based on both approaches, like the studies by Miyoshi [5] and Harasawa [6].

Limitations: Case series have suggested that hangekobokuto and hangeshashinto are effective for FD and that saireito, keihito, heiisan, and daikenchuto are effective for IBS. Their mechanisms of action are also well studied and account for their effects. For example, hangekobokuto was demonstrated to facilitate gastric emptying [10] and decrease the bowel gas [11] of patients with FD. However, so far, the effects of these Kampo medicines have not been evaluated in RCTs. Further studies are necessary to determine whether these Kampo medicines are really effective for FGIDs.

Recently, the gastroenterologists Tominaga and Arakawa published an excellent review article on Kampo for gastrointestinal disorders [69]. The strengths of their

Table 1 Beneficial effects of Kampo medicines on mind, stress, and body in functional gastrointestinal disorders

\begin{tabular}{llll}
\hline & Effects on brain & Effects on stress & Effects on GI tract \\
\hline Rikkunshito & $\begin{array}{l}\text { Reduces depressive symptoms and } \\
\text { high cortisol levels. }\end{array}$ & $\begin{array}{l}\text { Inhibits stress-induced activation } \\
\text { of HPA axis and SNS. }\end{array}$ & $\begin{array}{l}\text { Improves gastric motility dysfunction, } \\
\text { i.e., gastric emptying and gastric } \\
\text { accommodation. }\end{array}$ \\
& $\begin{array}{l}\text { Reduces anxiety. } \\
\text { Inhibits activities of CRH-producing } \\
\text { neurons. }\end{array}$ & & Modulates gastric hypersensitivity. \\
Keishikashakuyakuto & Has sedative, antidepressant-like & Inhibits stress-induced activation ghrelin secretion. \\
& effects. & of HPA axis. & Has anti-spasmodic, anti-inflammatory, \\
Increases BDNF & & and analgesic effects. \\
\hline
\end{tabular}

BDNF, brain-derived neurotrophic factor; $\mathrm{CRH}$, corticotropin-releasing hormone; Gl, gastrointestinal; HPA, hypothalamic-pituitary-adrenocortical; SNS, sympathetic nervous system. 
paper are that they reviewed the effects of Kampo on a broader range of disorders than we introduced in this article, including gastroesophageal reflux disorders and post-operative ileus, and that they described the basic mechanisms of action of Kampo on the gastrointestinal tract more precisely than we did. The strengths of our review article are that we introduced the RCTs and case series written in Japanese, to which Tominaga and Arakawa did not refer in their article, because we thought it important to introduce such pioneering works and that we described the effects of Kampo on extragastrointestinal tract symptoms and on the central nervous system and stress systems as well as on the gastrointestinal tract from the psychosomatic medicine point of view. Therefore, these two review articles will enable readers to understand the whole picture of the role of Kampo as a treatment for FGIDs.

\section{Conclusions}

This article reviewed the effects of Kampo medicines on FGIDs, focusing on FD and IBS. Four RCTs suggested the usefulness of rikkunshito for FD. Two RCTs suggested the usefulness of keishikashakuyakuto for IBS. These Kampo medicines act on both the brain and the gastrointestinal tract to alleviate subjective symptoms. Case series suggest that several other Kampo medicines are also effective for FGIDs. However, further studies are necessary to evaluate their efficacy.

\section{Competing interests}

The authors declare that they have no competing interests.

\section{Authors' contributions}

TO designed the study protocol, analyzed the data, and drafted the manuscript. HO reviewed "functional dyspepsia" and SN reviewed "irritable bowel syndrome". TI, SM, YK, and MM discussed the data and manuscript. All authors contributed to this project as members of Japanese Association of Oriental Psychosomatic Medicine, Evidence-Based Medicine Working Team (JOPM-EBM). All authors read and approved the final manuscript.

\section{Acknowledgements}

This work was supported in part by a Health Labour Sciences Research Grant for integrative medicine (H24-Iryo-Ippan-025 to TO). It was also conducted as part of the activities of the Japanese Association of Oriental Psychosomatic Medicine, Evidence-Based Medicine Working Team.

\section{Author details}

${ }^{1}$ Department of Psychosomatic Medicine, Graduate School of Medical Sciences, Kyushu University, Fukuoka 812-8582, Japan. ${ }^{2}$ Department of Medical Oncology, Division of Psychosomatic Medicine, Kinki University, Faculty of Medicine, Osaka 589-8511, Japan. ${ }^{3}$ Department of Psychosomatic Medicine, Japanese Red Cross Wakayama Medical Center, Komatsubara-dori 4-20, Wakayama 640-8558, Japan. ${ }^{4}$ Center of Japanese Oriental Medicine, Kashima Rosai Hospital, Labour Welfare Corporation, Doaihoncho 1-9108-2, Kamisu-shi, Ibaraki 314-0343, Japan. ${ }^{5}$ Department of Psychosomatic Medicine and Psychiatry, Gujo City Hospital, 1261 Shimadani, Hachiman-cho, Gujo City, Gifu 501-4222, Japan. 'Institute of Oriental Medicine, Tokyo Women's Medical University, 1-21-8 Tabata, Kita-ku, Tokyo 114-0014, Japan. ${ }^{7}$ Department of Psychosomatic Medicine, Nihon University Itabashi Hospital,

Oyaguchikamicho 30-1, Itabashi-ku, Tokyo 173-0032, Japan.
Received: 7 September 2013 Accepted: 30 December 2013

Published: 21 January 2014

\section{References}

1. Drossman DA: The functional gastrointestinal disorders and the Rome III process. Gastroenterology 2006, 130:1377-1390.

2. Levy RL, Olden KW, Naliboff BD, Bradley LA, Francisconi C, Drossman DA, Creed F: Psychosocial aspects of the functional gastrointestinal disorders. Gastroenterology 2006, 130:1447-1458.

3. Colin-Jones DG: Management of dyspepsia: report of a working party. Lancet 1988, 1:576-579.

4. Tack J, Talley NJ, Camilleri M, Holtmann G, Hu P, Malagelada JR, Stanghellini V: Functional gastroduodenal disorders. Gastroenterology 2006, 130:1466-1479.

5. Miyoshi A, Yachi A, Masamune O, Ishikawa M, Fukutomi H, Niwa H, Matsuo Y, Mori H, Tsuchiya M, Miwa T, et al: Mansei ien nadono huteino shoukakisyuuso ni taisuru TJ-43 Tsumura rikkunshito no rinsho hyoka -cisapride wo taishoyaku to shita tashisetsu hikaku shiken- (written in Japanese). Prog Med 1991, 11:1605-1631.

6. Harasawa S, Miyoshi A, Miwa T, Masamune O, Matsuo Y, Mori H, Nakazawa S, Suyama T, Hayakawa A, Nakashima M: Undo huzen gata no jofukubu huteishuuso (dysmotility-like dyspepsia) ni taisuru TJ-43 rikkunshito no tashisetsu kyodo shihango rinsho kenkyu (Double-blind multicenter post-marketing clinical trial of TJ-43 TSUMURA Rikkunshi-to for the dismotility like dyspepsia, written in Japanese). Igaku no Ayumi 1998, 187:207-229.

7. Harasawa S: NUD ni taisuru rikkunshito no yakuwari - tokuni dysmotility-like dyspepsia ni taisuru yuyousei ni tsuite (The role of rikkunshito in NUD, especially its usefulness for dysmotility-like dyspepsia, written in Japanese). Prog Med 1999, 19:843-848.

8. Tatsuta $M$, lishi $\mathrm{H}$ : Effect of treatment with liu-jun-zi-tang (TJ-43) on gastric emptying and gastrointestinal symptoms in dyspeptic patients. Aliment Pharmacol Ther 1993, 7:459-462.

9. Arai M, Matsumura T, Tsuchiya N, Sadakane C, Inami R, Suzuki T, Yoshikawa M, Imazeki F, Yokosuka O: Rikkunshito improves the symptoms in patients with functional dyspepsia, accompanied by an increase in the level of plasma ghrelin. Hepatogastroenterology 2012, 59:62-66.

10. Oikawa T, Ito G, Koyama H, Hanawa T: Prokinetic effect of a Kampo medicine, Hange-koboku-to (Banxia-houpo-tang), on patients with functional dyspepsia. Phytomedicine 2005, 12:730-734.

11. Oikawa T, Ito G, Hoshino T, Koyama H, Hanawa T: Hangekobokuto (Banxiahoupo-tang), a Kampo Medicine that Treats Functional Dyspepsia. Evid Based Complement Alternat Med 2009, 6:375-378.

12. Hoshino E: An old remedy for a new clinical entity:effect of a traditional Chinese herb recipe "rikkunshi-to" on non ulcer dyspepsia. Gastroenterology 1992, 102:A14.

13. Ooga M,HM: Jobu syoukakan shuuso ni taisuru Rikkunshito no yuuyousei - tannou shuushukukinou ijyou wo gappeisuru NUD deono kento (written in Japanese). Kampo lgaku 2001, 25:65-69.

14. Keohane J, Quigley EM: Functional dyspepsia: the role of visceral hypersensitivity in its pathogenesis. World J Gastroenterol 2006, 12:2672-2676.

15. Ochi M, Tominaga K, Tanaka F, Tanigawa T, Shiba M, Watanabe T, Fujiwara Y, Oshitani N, Higuchi K, Arakawa T: Effect of chronic stress on gastric emptying and plasma ghrelin levels in rats. Life Sci 2008, 82:862-868.

16. Geeraerts B, Vandenberghe J, Van Oudenhove L, Gregory L, Aziz Q, Dupont $P$, Demyttenaere $K$, Janssens J, Tack J: Influence of experimentally induced anxiety on gastric sensorimotor function in humans. Gastroenterology 2005, 129:1437-1444.

17. Van Oudenhove L, Vandenberghe J, Geeraerts B, Vos R, Persoons P, Demyttenaere K, Fischler B, Tack J: Relationship between anxiety and gastric sensorimotor function in functional dyspepsia. Psychosom Med 2007, 69:455-463.

18. Van Oudenhove L, Aziz Q: Recent insights on central processing and psychological processes in functional gastrointestinal disorders. Dig Liver Dis 2009, 41:781-787.

19. Oka T, Kanazawa F, Mine K, Nakagawa T, Maekawa T, Kokawa K, Miyake T: Studies of psychological factors affecting the prognosis of non-ulcer dyspepsia - Depressive state affects the prognosis of non-ulcer dyspepsia - (written in Japanese). Shinshin Iryo 1993, 5:1226-1232.

20. De la Roca-Chiapas JM, Solis-Ortiz S, Fajardo-Araujo M, Sosa M, CordovaFraga T, Rosa-Zarate A: Stress profile, coping style, anxiety, depression, 
and gastric emptying as predictors of functional dyspepsia: a case-control study. J Psychosom Res 2010, 68:73-81.

21. Miwa H, Watari J, Fukui H, Oshima T, Tomita T, Sakurai J, Kondo T, Matsumoto T: Current understanding of pathogenesis of functional dyspepsia. J Gastroenterol Hepatol 2011, 26(Suppl 3):53-60.

22. Harasawa S, Miwa T: Effect of TJ-43 TSUMURA Rikkunshito on gastric emptying and investigation of clinical trial (written in Japanese). Shokakika 1990, 12:215-212.

23. Kido T, Nakai Y, Kase Y, Sakakibara I, Nomura M, Takeda S, Aburada M: Effects of rikkunshi-to, a traditional Japanese medicine, on the delay of gastric emptying induced by N(G)-nitro-L-arginine. J Pharmacol Sci 2005, 98:161-167.

24. Tominaga K, Kido T, Ochi M, Sadakane C, Mase A, Okazaki H, Yamagami H, Tanigawa T, Watanabe K, Watanabe T, et al: The traditional Japanese medicine Rikkunshito promotes gastric emptying via the Antagonistic Action of the 5-HT3 receptor pathway in rats. Evid Based Complement Alternat Med 2011, 2011:248481.

25. Kusunoki H, Haruma K, Hata J, Ishii M, Kamada T, Yamashita N, Honda K, Inoue $\mathrm{K}$, Imamura H, Manabe $\mathrm{N}$, et al: Efficacy of Rikkunshito, a traditional Japanese medicine (Kampo), in treating functional dyspepsia. Intern Med 2010, 49:2195-2202.

26. Hayakawa T, Arakawa T, Kase Y, Akiyama S, Ishige A, Takeda S, Sasaki H, Uno H, Fukuda T, Higuchi K, Kobayashi K: Liu-Jun-Zi-Tang, a kampo medicine, promotes adaptive relaxation in isolated guinea pig stomachs. Drugs Exp Clin Res 1999, 25:211-218.

27. Kito Y, Suzuki H: Properties of Rikkunshi-to (TJ-43)-induced relaxation of rat gastric fundus smooth muscles. Am J Physiol Gastrointest Liver Physiol 2010, 298:G755-G763.

28. Yagi M, Homma S, Kubota M, linuma Y, Kanada S, Kinoshita Y, Ohtaki M, Yamazaki S, Murata H: The herbal medicine Rikkunshi-to stimulates and coordinates the gastric myoelectric activity in post-operative dyspeptic children after gastrointestinal surgery. Pediatr Surg Int 2004, 19:760-765.

29. Mochiki E, Yanai M, Ohno T, Kuwano H: The effect of traditional Japanese medicine (Kampo) on gastrointestinal function. Surg Today 2010, 40:1105-1111.

30. Yanai M, Mochiki E, Ogawa A, Morita H, Toyomasu Y, Ogata K, Tabe Y, Ando $H, O h n o ~ T$, Asao $T$, et al: Intragastric administration of rikkunshito stimulates upper gastrointestinal motility and gastric emptying in conscious dogs. J Gastroenterol 2013, 48:611-619.

31. Shiratori M, Shoji T, Kanazawa M, Hongo M, Fukudo S: Effect of rikkunshito on gastric sensorimotor function under distention. Neurogastroenterol Motil 2011, 23:323-329. e155-156.

32. Hattori T: Rikkunshito and ghrelin. Int J Pept 2010, 2010:283549.

33. Takeda H, Sadakane C, Hattori T, Katsurada T, Ohkawara T, Nagai K, Asaka M: Rikkunshito, an herbal medicine, suppresses cisplatin-induced anorexia in rats via 5-HT2 receptor antagonism. Gastroenterology 2008, 134:2004-2013.

34. Takeda H, Muto S, Nakagawa K, Ohnishi S, Sadakane C, Saegusa Y, Nahata M, Hattori T, Asaka M: Rikkunshito as a ghrelin enhancer. Methods Enzymol 2012, 514:333-351.

35. Matsumura T, Arai M, Yonemitsu Y, Maruoka D, Tanaka T, Suzuki T, Yoshikawa M, Imazeki F, Yokosuka O: The traditional Japanese medicine Rikkunshito increases the plasma level of ghrelin in humans and mice. J Gastroenterol 2010, 45:300-307.

36. Takiguchi S, Hiura Y, Takahashi T, Kurokawa Y, Yamasaki M, Nakajima K, Miyata H, Mori M, Hosoda H, Kangawa K, Doki Y: Effect of rikkunshito, a Japanese herbal medicine, on gastrointestinal symptoms and ghrelin levels in gastric cancer patients after gastrectomy. Gastric Cancer 2013, 16:167-174.

37. Fujitsuka N, Asakawa A, Uezono Y, Minami K, Yamaguchi T, Niijima A, Yada T, Maejima Y, Sedbzar U, Sakai T, Hattori T, Kase Y, Inui A: Potentiation of ghrelin signaling attenuates cancer anorexia-cachexia and prolongs survival. Transl Psychiatry 2011, 1:e23

38. Oka T, Nakagawa T, Matsuura T, Kokawa K: Jofukubu hutei shuso ni taisuru TJ-43 TSUMURA rikkunshito no kouka no kento (Efficacy of TJ-43 TSUMURA Rikkunshito on patients with upper gastrointestinal nonspecific symptoms: Focusing on antidepressant effect, written in Japanese). Jpn J Clin Exp Med 1990, 67:243-245.

39. Oka T: Studies on the effects of rikkunshi-to and hochu-ekki-to in adrenal and autonomic nervous functions (written in Japanese). Jpn J Oriental Med 1993, 43:439-446.

40. Naito $T$, Itoh $H$, Takeyama M: Some gastrointestinal function regulatory Kampo medicines have modulatory effects on human plasma adrenocorticotropic hormone and cortisol levels with continual stress exposure. Biol Pharm Bull 2003, 26:101-104.

41. Sato $Y$, Katagiri F, Itoh $H$, Takeyama M: Effects of some kampo medicines on plasma levels of neuropeptide $Y$ under venipuncture stress. Biol Pharm Bull 2005, 28:1757-1761.

42. Tomokane M, Tashiro S: Effect of odor of Kampo medicine, its pharmacokinetics and interaction with modern medicine drugs. Methods Kampo Pharmacol 1997, 1:110-116.

43. Saegusa Y, Takeda H, Muto S, Nakagawa K, Ohnishi S, Sadakane C, Nahata M, Hattori T, Asaka M: Decreased plasma ghrelin contributes to anorexia following novelty stress. Am J Physiol Endocrinol Metab 2011, 301:E685-696.

44. Functional dyspepsia. In A Guideline for the Diagnosis and Treatment of Psychosomatic Diseases. Edited by Komaki G, Kubo C, Fukudo S. Tokyo: Igaku shoin; 2006:42-62.

45. Kawahara H, Kubota A, Hasegawa T, Okuyama H, Ueno T, Ida S, Fukuzawa M: Effects of rikkunshito on the clinical symptoms and esophageal acid exposure in children with symptomatic gastroesophageal reflux. Pediatr Surg Int 2007, 23:1001-1005.

46. Tominaga K, Iwakiri R, Fujimoto K, Fujiwara Y, Tanaka M, Shimoyama Y, Umegaki E, Higuchi K, Kusano M, Arakawa T: Rikkunshito improves symptoms in PPI-refractory GERD patients: a prospective, randomized, multicenter trial in Japan. J Gastroenterol 2012, 47:284-292.

47. Kawahara H, Mitani Y, Nomura M, Nose K, Yoneda A, Hasegawa T, Kubota A, Fukuzawa M: Impact of rikkunshito, an herbal medicine, on delayed gastric emptying in profoundly handicapped patients. Pediatr Surg Int 2009, 25:987-990

48. Seike J, Sawada T, Kawakita N, Yamamoto Y, Yuasa Y, Yamai H, Takachi H, Yoshida T, Tangoku A: A New Candidate Supporting Drug, Rikkunshito, for the QOL in Advanced Esophageal Cancer Patients with Chemotherapy Using Docetaxel/5-FU/CDDP. Int I Surg Oncol 2011, 2011:715623.

49. Ohno T, Yanai M, Ando H, Toyomasu Y, Ogawa A, Morita H, Ogata K, Mochiki E, Asao T, Kuwano H: Rikkunshito, a traditional Japanese medicine, suppresses cisplatin-induced anorexia in humans. Clin Exp Gastroenterol 2011, 4:291-296.

50. Oka T, Tamagawa Y, Hayashida S, Kaneda Y, Kodama N, Tsuji S: Rikkunshi-to attenuates adverse gastrointestinal symptoms induced by fluvoxamine. Biopsychosoc Med 2007, 1:21

51. Mizuno S, Nagata K, Yoshida K, Sasaki H, Kojo H, Miura K, Tamura N, Hirayama S, Nakagawa T, Inoue M, Ogawa N: Kabinsei Cho Shoukougun ni taisuru keishikashakuyakuto ekisu no chiryo kouka - mepenzolate bromide tono hikaku shiken - (witten in Japanese). Shindan to Chiryo 1985, 73:1143-1152.

52. Sasaki D, Uehara A, Hiwatashi N, Sekiguchi T, Nakahara A, Nakai Y, Mizuno M, Morita N, Namiki M, Nakano S: Kabinsei Cho Shoukougun ni taisuru keishikashakuyakuto no rinsho kouka - tashisetsu kyodo musakui waritsuke gunkan hikaku rinsho shiken - (witten in Japanese). Jpn J Clin Exp Med 1998, 75:1136-1152.

53. Oka T, Mine K, Nakagawa T: Studies on Kampo therapy for patients with irritable bowel syndrome who were not successfully treated with keishi-ka-shanuyaku-to - the usefulness of taking extracolonic symptoms into account - (written in Japanese). J Jpn Assoc Oriental Psychosom Med 1993, 8:37-44

54. Maeda T, Shinozuka K, Baba K, Hayashi M, Hayashi E: Effect of shakuyakukanzoh-toh, a prescription composed of shakuyaku (Paeoniae Radix) and kanzoh (Glycyrrhizae Radix) on guinea pig ileum. J Pharmacobiodyn 1983, 6:153-160.

55. Ai M, Yamaguchi T, Odaka T, Mitsuhashi K, Shishido T, Yan J, Seza A, Saisho H: Objective assessment of the antispasmodic effect of shakuyaku-kanzo-to (TJ-68), a Chinese herbal medicine, on the colonic wall by direct spraying during colonoscopy. World J Gastroenterol 2006, 12:760-764.

56. Takagi K, Harada M: Pharmacological studies on herb paeony root III. Effects of paeoniflorin on circulatory and respiratory systems and isolated organs. Yakugaku Zasshi 1969, 89:893-898.

57. Hayashi M, Baba K, Maeda T: Effect of shakuyaku-extract on electrical stimulation induced contraction of guinea pig ileum. Yakugaku Zasshi 1990, 110:139-143.

58. Tsuji S, Yasuda K, Sumi G, Cho H, Tsuzuki T, Okada H, Kanzaki H: Shakuyakukanzo-to inhibits smooth muscle contractions of human pregnant uterine tissue in vitro. J Obstet Gynaecol Res 2012, 38:1004-1010. 
59. Takagi K, Harada M: Pharmacological studies on herb paeony root II. Anti-inflammatory effect, inhibitory effect on gastric juice secretion, preventive effect on stress ulcer, antidiuretic effect of paeoniflorin and combined effects with licorice component FM100. Yakugaku Zasshi 1969, 89:887-892.

60. Takagi K, Harada M: Pharmacological studies on herb paeony root I. Central effects of paeoniflorin and combined effects with licorice component FM100. Yakugaku Zasshi 1969, 89:879-886.

61. Mao QQ, Ip SP, Tsai SH, Che CT: Antidepressant-like effect of peony glycosides in mice. J Ethnopharmacol 2008, 119:272-275.

62. Mao QQ, Ip SP, Ko KM, Tsai SH, Xian YF, Che CT: Effects of peony glycosides on mice exposed to chronic unpredictable stress: further evidence for antidepressant-like activity. J Ethnopharmacol 2009, 124:316-320.

63. Mao QQ, Ip SP, Ko KM, Tsai SH, Che CT: Peony glycosides produce antidepressant-like action in mice exposed to chronic unpredictable mild stress: effects on hypothalamic-pituitary-adrenal function and brain-derived neurotrophic factor. Prog Neuropsychopharmacol Biol Psychiatry 2009, 33:1211-1216.

64. Mao QQ, Xian YF, Ip SP, Tsai SH, Che CT: Long-term treatment with peony glycosides reverses chronic unpredictable mild stress-induced depressive-like behavior via increasing expression of neurotrophins in rat brain. Behav Brain Res 2010, 210:171-177.

65. Mao QQ, Ip SP, Xian YF, Hu Z, Che CT: Anti-depressant-like effect of peony: a mini-review. Pharm Biol 2012, 50:72-77.

66. Mao QQ, Huang Z, Ip SP, Xian YF, Che CT: Peony glycosides reverse the effects of corticosterone on behavior and brain BDNF expression in rats. Behav Brain Res 2012, 227:305-309.

67. Whorwell PJ, McCallum M, Creed FH, Roberts CT: Non-colonic features of irritable bowel syndrome. Gut 1986, 27:37-40

68. Tilbe K, Sullivan S: The extracolonic manifestations of the irritable bowel syndrome. CMAJ 1990, 142:539-540.

69. Tominaga K, Arakawa T: Kampo medicines for gastrointestinal tract disorders: a review of basic science and clinical evidence and their future application. J Gastroenterol 2013, 48:452-462.

doi:10.1186/1751-0759-8-5

Cite this article as: Oka et al.: Effects of Kampo on functional gastrointestinal disorders. BioPsychoSocial Medicine 2014 8:5.

\section{Submit your next manuscript to BioMed Central and take full advantage of:}

- Convenient online submission

- Thorough peer review

- No space constraints or color figure charges

- Immediate publication on acceptance

- Inclusion in PubMed, CAS, Scopus and Google Scholar

- Research which is freely available for redistribution 